\title{
Tribute to Professor José Simões Redinha on the occasion of his 80th birthday
}



This special issue of the Journal of Chemical Thermodynamics is dedicated to Retired Professor José Simões Redinha: a Scientist, a Teacher, a Mentor.

Professor J. Simões Redinha was born on 19 October 1927 in Campizes-Condeixa, a small town in the vicinity of Coimbra, the major city of the central region of Portugal. He finished his high school education in this city in 1947, and started to study Chemistry in the University of Coimbra in the same year. In 1951, he obtained his first degree in Physical Chemical Sciences with the excellent grade of 17/20. Recognized for his excellent academic talent, just after obtaining his first degree, José Simões Redinha was invited to become a member of the teaching staff of the Chemistry Section in the Faculty of Science of Coimbra University.
Between 1956 and 1958 he was a Ph.D student at the Imperial College of Science and Technology of the London University, under the supervision of Professor J.A. Kitchener. Professor José Simões Redinha obtained his Ph.D. in 1958 with the thesis entitled "A study of the thermodynamics of cation-exchange reactions on polystyrene sulphonate resins", which marked the beginning of a scientific career dedicated to Chemical Thermodynamics. He has kept close links with Imperial College, where he was Visiting Professor in 1971 and 1978.

In 1964, he was made Full Professor of the University of Coimbra, following unanimous approval in the public examinations for this position. 
Professor Simões Redinha's research activities have been devoted to two main topics: the study of the structure of non-electrolyte solutions and the investigation of polymorphism of organic compounds, using thermodynamic properties, complemented by data from other methods, such as infrared spectroscopy and computational calculations.

The study of non-electrolyte solutions was the original aim of J. Simões Redinha's research. He was particularly interested in the role of water as a solvent, on the nature of the interaction of non-polar groups with this solvent and on the influence of the number and relative position of polar groups on solvation. Solvation enthalpies, obtained from solution and vaporization/sublimation calorimetric measurements, were determined for solutes carefully chosen to mimic different molecular arrangements in water and in selected organic solvents. These were then used to seek explanations to the above questions. The molecular interpretation of macroscopic thermodynamic properties was accomplished by taking into account the different partial contributions to the solvation process, and subsequently performing quantitative determination of solute-solvent interactions. Using this procedure, by combining solvation thermodynamic data and statistical mechanical calculations, he demonstrated that the apparently anomalous solvation enthalpies in water relative to organic solvents were a result of a smaller water molecular diameter and a lower number density. When these differences are taken into account and the solute-solvent interaction term is calculated, no anomalies are observed.

For polyfunctional solutes, he recognized that the change in solute conformation on going from the gas phase into the solution could give rise to a non-negligible contribution to solvation properties. He was a pioneer in proposing and calculating this contribution, using computational methods, both to obtain a correct interpretation of solvation enthalpy, and also to discuss the interaction forces in the solid and liquid states, based on sublimation/vaporization enthalpic data.

Because of Professor Redinha's qualities as a diligent teacher and eminent scientist, several students, particularly from the Faculty of Pharmacy in Coimbra, have chosen to carry out their postgraduate studies in his research group. The scientific area of the group has consequently been expanded to include the study of the polymorphism of organic compounds, in response to the needs of this area. He has been interested in the characterization of the polymorphs of solids used as pharmaceuticals, applying differential scanning calorimetry, thermo-microscopy, infrared spectroscopy, X-ray diffraction, etc. Moreover, since crystallization from solvents is one of the principal ways of obtaining different poly- morphic forms, he has made use of his expertise in the interactions in solution to understand the effect of solvents on the solid form obtained.

J. Simões Redinha is author of more than 80 scientific publications, and was the mentor of 25 post-graduate theses. Although José Simões Redinha has devoted most of his life to research, he also had an extensive participation in the University life. He was Director of the Faculty of Science of the University of Coimbra (1972-1974) and Head of the Chemistry Department at various times. The introduction of the Engineering degrees in the newly created Faculty of Science and Technology was a major achievement during his directorship. Some years previously (1963-1966) he accepted the invitation to establish, equip and direct the Chemistry Section of the University of Lourenço Marques (today Maputo) in Mozambique. More recently, he represented Portugal in EURATOM (1990-1993) and in EURACHEM (1990-1996). He has also been made a member of the Portuguese Academy of Science.

He has maintained a remarkable and constant concern in keeping a close link between the University and the economic and social activities of the country. He has always accepted a new practical problem as a challenge and made use of his vast, solid culture on chemistry to look for solutions. To respond to the needs of the local society and industry, he organized and directed a laboratory in the Chemistry Department for several years devoted to these problems, and in 1992, was able to set up a non profit making laboratory association, ÆMITEQ, with modern laboratories, which is devoted to meeting industrial and other needs in analytical chemistry in the central region of Portugal, which he directed until 2002.

Professor Redinha is a multitalented and dynamic personality. Although formally retired ten years ago, at the age of 70 , he has continued his research activities with the same commitment and dedication as ever, advising, supporting, innovating research in chemical thermodynamics. This special issue is the statement of our admiration and gratitude for the scientific and personal enrichment which we have gained in our daily contact with him. We congratulate Prof. Redinha for his 80th birthday and express our best wishes for the future.

M. Ermelinda S. Eusébio

Chemistry Department, University of Coimbra, Portugal

João Canotilho

Faculty of Pharmacy, University of Coimbra, Portugal

Available online 31 March 2007 